\title{
Normalization and microbial differential abundance strategies depend upon data characteristics
}

Sophie Weiss ${ }^{1}$, Zhenjiang Zech Xu², Shyamal Peddada ${ }^{3}$, Amnon Amir ${ }^{2}$, Kyle Bittinger ${ }^{4}$, Antonio Gonzalez², Catherine Lozupone ${ }^{5}$, Jesse R. Zaneveld ${ }^{6}$, Yoshiki Vázquez-Baeza, Amanda Birmingham ${ }^{8}$, Embriette R. Hyde ${ }^{2}$ and Rob Knight ${ }^{2,7,9^{*}}$

\begin{abstract}
Background: Data from $16 \mathrm{~S}$ ribosomal RNA (rRNA) amplicon sequencing present challenges to ecological and statistical interpretation. In particular, library sizes often vary over several ranges of magnitude, and the data contains many zeros. Although we are typically interested in comparing relative abundance of taxa in the ecosystem of two or more groups, we can only measure the taxon relative abundance in specimens obtained from the ecosystems. Because the comparison of taxon relative abundance in the specimen is not equivalent to the comparison of taxon relative abundance in the ecosystems, this presents a special challenge. Second, because the relative abundance of taxa in the specimen (as well as in the ecosystem) sum to 1, these are compositional data. Because the compositional data are constrained by the simplex (sum to 1) and are not unconstrained in the Euclidean space, many standard methods of analysis are not applicable. Here, we evaluate how these challenges impact the performance of existing normalization methods and differential abundance analyses.
\end{abstract}

Results: Effects on normalization: Most normalization methods enable successful clustering of samples according to biological origin when the groups differ substantially in their overall microbial composition. Rarefying more clearly clusters samples according to biological origin than other normalization techniques do for ordination metrics based on presence or absence. Alternate normalization measures are potentially vulnerable to artifacts due to library size.

Effects on differential abundance testing: We build on a previous work to evaluate seven proposed statistical methods using rarefied as well as raw data. Our simulation studies suggest that the false discovery rates of many differential abundance-testing methods are not increased by rarefying itself, although of course rarefying results in a loss of sensitivity due to elimination of a portion of available data. For groups with large $(\sim 10 \mathrm{x})$ differences in the average library size, rarefying lowers the false discovery rate. DESeq2, without addition of a constant, increased sensitivity on smaller datasets ( $<20$ samples per group) but tends towards a higher false discovery rate with more samples, very uneven $(\sim 10 x)$ library sizes, and/or compositional effects. For drawing inferences regarding taxon abundance in the ecosystem, analysis of composition of microbiomes (ANCOM) is not only very sensitive (for $>20$ samples per group) but also critically the only method tested that has a good control of false discovery rate.

Conclusions: These findings guide which normalization and differential abundance techniques to use based on the data characteristics of a given study.

Keywords: Microbiome, Normalization, Differential abundance, Statistics

\footnotetext{
* Correspondence: robknight@ucsd.edu

${ }^{2}$ Departments of Pediatrics, University of California San Diego, 9500 Gilman

Drive, MC 0763, La Jolla, CA 92093, USA

${ }^{7}$ Department of Computer Science \& Engineering, University of California San

Diego, La Jolla, CA 92093, USA

Full list of author information is available at the end of the article
} 


\section{Background}

Although data produced by high-throughput sequencing has been proven extremely useful for understanding microbial communities, the interpretation of these data is complicated by several statistical challenges. Following initial quality control steps to account for errors in the sequencing process, microbial community sequencing data is typically organized into large matrices where the columns represent samples, and the rows contain observed counts of clustered sequences commonly known as operational taxonomic units, or OTUs, that represent bacteria types. These tables are often referred to as OTU tables. Several features of OTU tables can cause erroneous results in downstream analyses if unaddressed. First, the microbial community in each biological sample may be represented by very different numbers of sequences (i.e., library sizes), reflecting differential efficiency of the sequencing process rather than true biological variation. This problem is exacerbated by the observation that the full range of species is rarely saturated, so that more bacterial species are observed with more sequencing (similar trends by sequencing depth hold for discovery of genes in shotgun metagenomic samples [1, 2]). Thus, samples with relatively few sequences may have inflated beta ( $\beta$, or between sample) diversity, since authentically shared OTUs are erroneously scored as unique to samples with more sequences [3]. Second, most OTU tables are sparse, meaning that they contain a high proportion of zero counts ( 90\%) [4]. This sparsity implies that the counts of rare OTUs are uncertain, since they are at the limit of sequencing detection ability when there are many sequences per sample (i.e., large library size) and are undetectable when there are few sequences per sample. Third, the total number of reads obtained for a sample does not reflect the absolute number of microbes present, since the sample is just a fraction of the original environment. Since the relative abundances sum to 1 and are non-negative, the relative abundances represent compositional data [5-7]. Compositional data are constrained by the simplex (sum to 1) and are not free floating in the Euclidean space; therefore, standard methods of analysis are not applicable. For example, an increase in abundance of one prevalent bacterial taxon can lead to spurious negative correlations for the abundance of other taxa [8]. Uneven sampling depth, sparsity, and the fact that researchers are interested in drawing inferences on taxon abundance in the ecosystem using the specimen level data represent serious challenges for interpreting data from microbial survey studies.

In an attempt to mitigate some of these three challenges and aid in data interpretation, data are often normalized by various computational processes prior to downstream analysis. Normalization is the process of transforming the data in order to enable accurate comparison of statistics from different measurements by eliminating artifactual biases in the original measurements. For example, in microbiome data, biases that reflect no true difference in underlying biology can exist due to variations in sample collection, library preparation, and/or sequencing and can manifest as, e.g., uneven sampling depth and sparsity. After effective normalization, data from different samples can then be compared to each other. Ordination analysis, such as principal coordinate analysis (PCoA) [9], is often then applied to these normalized data to visualize broad trends of how similar or different bacterial populations are in certain sample types, such as healthy vs. sick patients (ordination is a general term for a family of techniques that summarize and project multivariate community data into lower-dimension space). This enables easy visual inspection of sample groupings, driven by sample bacterial content similarity/dissimilarity, and any association with sample metadata. Researchers may further wish to determine, through statistical testing, which specific bacteria are significantly differentially abundant between two ecosystems; this process is known as differential abundance testing. Significant changes in certain bacterial species abundances are linked to inflammatory bowel diseases [10], diarrhea [11], obesity [12-14], HIV [15], diet [16], culture, age, and antibiotic use [17], among many other conditions. However, the reliability of these findings depends upon how much the statistical challenges posed by the underlying community sequence data impact the chosen normalization and differential abundance testing techniques.

This paper therefore examines how various normalization and differential abundance testing procedures available in the literature are affected by the challenges inherent in microbiome data. Recent work in this area [18] addresses the performance of parametric normalization and differential abundance testing approaches for microbial ecology studies, but it is primarily focused on estimating proportions. We update and expand those findings using both real and simulated datasets exemplifying the challenges noted above.

\section{Normalization approaches}

Because normalization is intended to enable meaningful comparison of data from different measurements, it is critical to the validity of all downstream analyses. Microbial ecologists in the era of high-throughput sequencing have commonly normalized their OTU matrices by rarefying or drawing without replacement from each sample such that all samples have the same number of total counts. This process, in effect, standardizes the library size across samples, mitigating the first challenge discussed above. Samples with total counts below the defined threshold are excluded, sometimes leading researchers to face difficult trade-offs between sampling depth and the number of samples evaluated. To ensure an informative total sum is 
chosen, rarefaction curves can be constructed [19]. These curves plot the number of counts sampled (rarefaction depth) vs. the expected value of species diversity. Rarefaction curves provide guidance that allows users to avoid gutting the species diversity found in samples by choosing too low a rarefaction depth. The origins of rarefying sample counts are mainly in-sample species diversity measures or alpha diversity $[19,20]$. However, more recently rarefying has been used in the context of $\beta$-diversity $[21,22]$. Rarefying samples for normalization is now the standard in microbial ecology and is present in all major data analysis toolkits for this field [23-26]. While rarefying is not an ideal normalization method, as it potentially reduces statistical power depending upon how much data is removed and does not address the challenge of compositional data, alternatives to rarefying have not been sufficiently developed until recently.

Another common normalization method besides rarefying is scaling. Scaling refers to multiplying the matrix counts by fixed values or proportions, i.e., scale factors, and specific effects of scaling methods depend on the scaling factors chosen and how they are applied. Often, a particular quantile of the data is used for normalization, but choosing the most effective quantile is difficult [4, 27-30]. Furthermore, while microbiome data are frequently sparse as discussed above, scaling can overestimate or underestimate the prevalence of zero fractions, depending on whether zeros are left in or thrown out of the scaling $[8,31]$. This is because putting all samples of varying sampling depth on the same scale ignores the differences in sequencing depth (and therefore resolution of species), caused by differing library sizes between the samples. For example, a rare species having zero counts in a small library size sample can have fractional abundance in a large library size sample (unless further mathematical modeling beyond simple total sum scaling, or proportions, is applied). Scaling can also distort OTU correlations across samples, again due to zeros and differences in sequencing depth [5, 6, 8, 32, 33].

A further alternative is Aitchison's log-ratio transformation [5], which is applicable to compositional data. However, because the log transformation cannot be applied to zeros (which are often well over half of microbial data counts [4]), sparsity can be problematic for methods that rely on this transformation. One approach to this issue is to replace zeros with a small value, known as a pseudocount [7]. While numerous papers discuss the choice of pseudocount values [34-37], which can influence results, there is no clear consensus on how to choose them. A Bayesian formulation to the problem is available in the literature [38]; however, this formulation assumes a Dirichlet-multinomial framework, which imposes a negative correlation structure on every pair of taxa $[7,39]$.

\section{Differential abundance testing methods}

For OTU differential abundance testing between groups (e.g., case vs. control), a common approach is to first rarify the count matrix to a fixed depth and then apply a nonparametric test (e.g., the Mann-Whitney/Wilcoxon rank-sum test for tests of two groups; the Kruskal-Wallis test for tests of multiple groups). Nonparametric tests are often preferred because OTU counts are not exactly normally distributed [40]. However, when analyzing relative abundance data, this approach does not account for the fact that the relative abundances are compositional. Also, nonparametric tests such as the Kruskal-Wallis test do not fare well in terms of power when sample size is small and/or the data are sparse [4]. Recently, promising parametric models that make stronger assumptions about the data have been developed in the subfields of transcriptomics ("RNA-Seq") and metagenomic sequencing. These may additionally be useful for microbial marker gene data $[4,18,27,30,41-44]$. Such models have greater power if their assumptions about the data are correct; however, studies of these models on RNASeq data have shown that they can yield a high level of false negatives or false positives when relevant assumptions are not valid [45].

These parametric models are composed of a generalized linear model (GLM) that assumes a distribution [46], and the choice of distribution is often debatable [4, 18, 45, 47-53]. To allow for extra Poisson variation in the count data, often the Poisson parameter is modeled by a gamma distribution so that the marginal count distribution is negative binomial (NB) [27, 30, 41]. Although the NB model allows for extra Poisson variation, it does not fit the data well when there are many zeros $[4,49]$. Zero-inflated GLMs, the most promising of which is the zero-inflated lognormal, attempt to overcome this limitation [4]. The zero-inflated lognormal tries to address sparsity and unequal sampling depth (library size) by separately modeling "structural" zero counts generated by, e.g., under-sequencing and zeros generated by the biological distribution of taxa, while the non-zero counts are modeled by the lognormal distribution.

\section{Results and discussion Normalization efficacy}

Proper normalization may potentially remove biases and variations introduced by the sampling and sequencing process, so that the normalized data reflects the underlying biology. One way to evaluate whether normalization has effectively removed such biases is to examine whether the groupings of normalized data after ordination analysis reflect biological or artifactual features. For example, Fig. 1a shows a PCoA plot based on normalized data, which demonstrates that the subjects are matched to the keyboard they touched, and samples from the same 
a

PERMANOVA $\mathrm{R}^{2}$

Sequences per sample: 0

Subject_ID: 0.30*

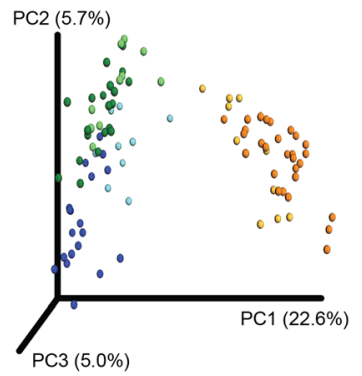

b

PERMANOVA $R^{2}$

Sequences per sample: $0.12^{*}$

Subject_ID: $0.21^{*}$

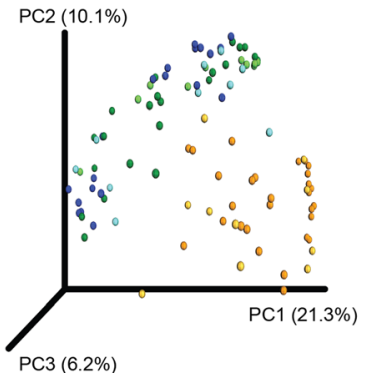

c

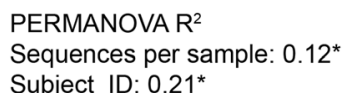

Subject ID: $0.21^{*}$

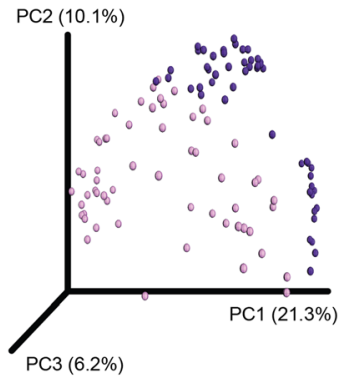

50 sequences per sample

500 sequences per sample

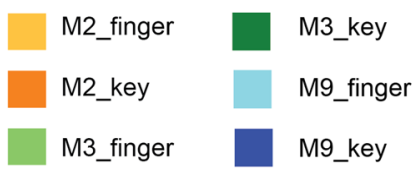

Fig. 1 Normalization is critical to result interpretation. a The forensic study matching subject's fingers to the keyboards they touched (Fierer et al.), rarefied at 500 sequences per sample. b, c Data not normalized, with a random half of the samples subsampled to 500 sequences per sample and the other half to 50 sequences per sample. b Colored by subject_ID. c Colored by sequences per sample. Nonparametric ANOVA (PERMANOVA) $R^{2}$ roughly represents the percent variance that can be explained by the given variable. Asterisk ${ }^{*}$ ) indicates significance at $p<0.01$. The distance metric of unweighted UniFrac was used for all panels

subject are close together in PCoA space [54]. With the UniFrac distance metric used here, such close sample grouping indicates similar bacterial communities. However, the grouping becomes less distinguishable when the data is not normalized (Fig. 1b), and this is potentially caused by the artifact of sequence depth variation (Fig. 1c). The highly sequenced samples appear more similar to each other than the shallowly sequenced samples because the highly sequenced samples are scored as sharing the same rare taxa (it should be noted that when groupings in a PCoA plot do not appear to reflect artifactual features, this does not necessarily indicate that they reflect relevant biology, because it is trivial to generate data with no biological signal that nonetheless form or appear to form clusters).

To assess the seven proposed normalization methods shown in Table 1, we first examined prior simulations [18]. Briefly, only necessary modifications ("Methods" section) were made to the code of McMurdie and Holmes [18], making our approach easily comparable. If all techniques are run on the same samples as those used when rarefying, the rarefying technique clusters as many samples into their biological groupings as the alternatives (Fig. 2). If low-depth samples are not dropped from analysis for methods other than rarefying, this could impact clustering by biological origin, especially for presence/absence distance metrics (Additional file 1: Figure 1S). This practice of removing low-depth samples from the analysis is supported by the recent discovery that small biomass samples are of poorer quality and contain a higher proportion of contaminating sequences $[55,56]$. Furthermore, alternatives to rarefying also recommend discarding lowdepth samples, particularly if they cluster separately from the rest of the data $[4,42]$. These results demonstrate that previous microbiome ordinations using rarefying as a normalization method likely clustered similarly compared to newer techniques, especially if some low-depth samples were removed.

For unweighted metrics that are based on species presence and absence, like binary Jaccard and unweighted UniFrac, the variance-stabilizing transformation performed by DESeq clusters many fewer samples according to biological origin than other techniques. This is because, as done in McMurdie and Holmes [18]), the negative values resulting from the log-like transformation are set to zero, causing the method to ignore many rare species completely. No good solution currently exists for the negative value output by the DESeq technique. DESeq was developed mainly for use with Euclidean metrics $[57,58]$, for which negative values are not a problem; however, this issue yields misleading results for ecologically useful non-Euclidean measures, like Bray-Curtis [59] dissimilarity. Also, the negative values pose a problem to the branch length of UniFrac $[57,58]$. The alternative to setting the negative values to zero, which is adding the absolute value of the lowest negative value back to the normalized matrix, will not work with 
Table 1 Normalization methods investigated in this study

\begin{tabular}{|c|c|}
\hline Method & Description \\
\hline None & No correction for unequal library sizes is applied \\
\hline Proportion & Counts in each column are scaled by the column's sum \\
\hline Rarefy & Each column is subsampled to even depth without replacement (hypergeometric model) \\
\hline logUQ & $\begin{array}{l}\text { Log upper quartile-Each sample is scaled by the 75th percentile of its count distribution; then, } \\
\text { the counts are log transformed }\end{array}$ \\
\hline CSS & $\begin{array}{l}\text { Cumulative sum scaling-This method is similar to logUQ, except that CSS enables a flexible sample } \\
\text { distribution-dependent threshold for determining each sample's quantile divisor. Only the segment } \\
\text { of each sample's count distribution that is relatively invariant across samples is scaled by CSS. This } \\
\text { attempts to mitigate the influence of larger count values in the same matrix column }\end{array}$ \\
\hline DESeqVS & $\begin{array}{l}\text { Variance stabilization (VS)_For each column, a scaling factor for each OTU is calculated as that } \\
\text { OTU's value divided by its geometric mean across all samples. All of the reads for each column } \\
\text { are then divided by the median of the scaling factors for that column. The median is chosen to } \\
\text { prevent OTUs with large count values from having undue influence on the values of other OTUs. } \\
\text { Then, using the scaled counts for all the OTUs and assuming a Negative Binomial (NB) distribution } \\
\text { a mean-variance relation is fit. This adjusts the matrix counts using a log-like transformation in the } \\
\text { NB generalized linear model (GLM) such that the variance in an OTU's counts across samples is } \\
\text { approximately independent of its mean }\end{array}$ \\
\hline edgeR-TMM & $\begin{array}{l}\text { Trimmed Mean by M-Values (TMM)_-The TMM scaling factor is calculated as the weighted mean } \\
\text { of log-ratios between each pair of samples, after excluding the highest count OTUs and OTUs } \\
\text { with the largest log-fold change. This minimizes the log-fold change between samples for most } \\
\text { OTUs. The TMM scaling factors are usually around 1, since TMM normalization, like DESeqVS, } \\
\text { assumes that the majority of OTUs are not differentially abundant. The normalization factors } \\
\text { for each sample are the product of the TMM scaling factor and the original library size }\end{array}$ \\
\hline
\end{tabular}

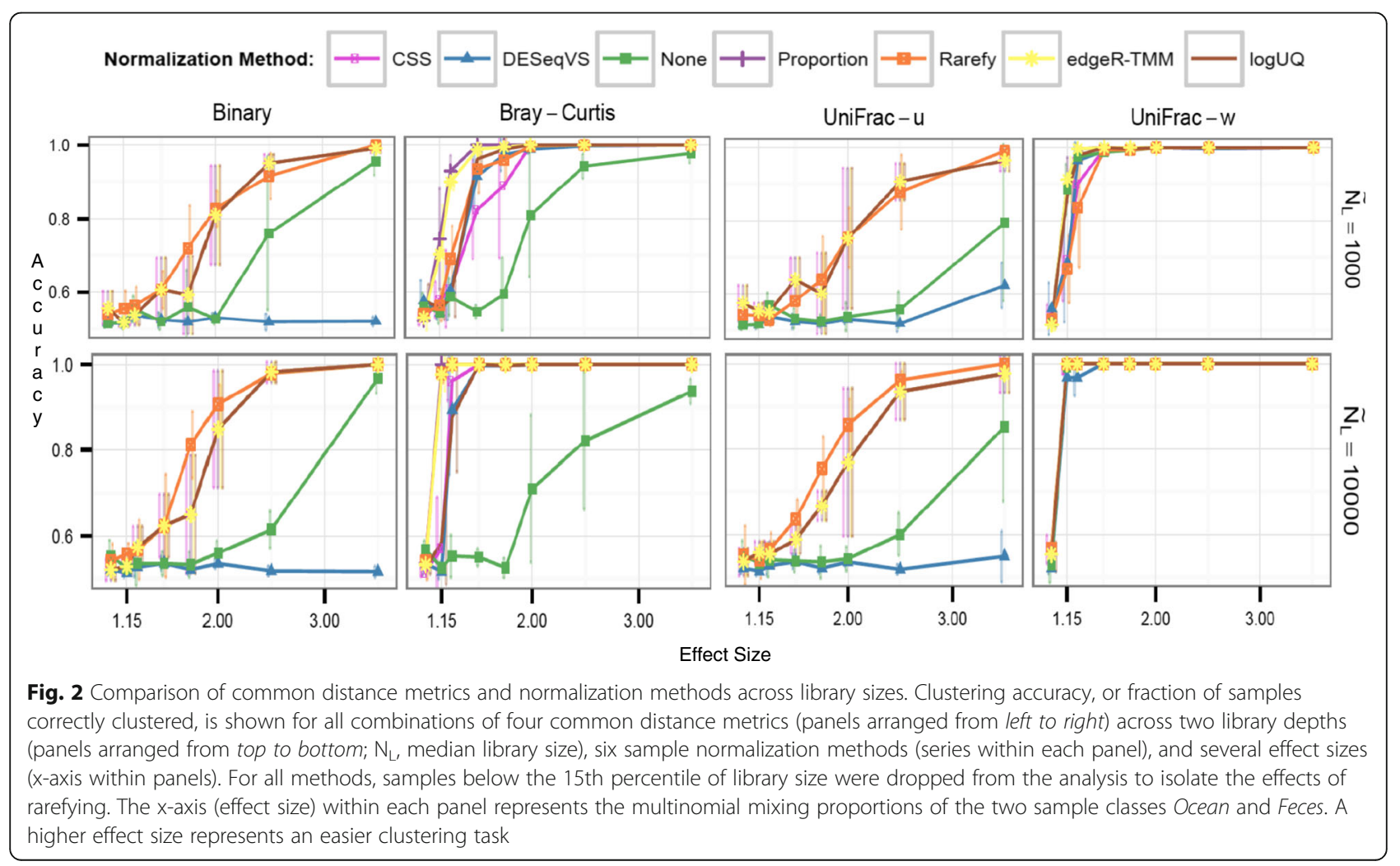


distance metrics that are not Euclidean because it amounts to multiplying the original matrix by a constant due to the log-like transformation of DESeq [28]. Also, the addition of a constant (or pseudocount; here, one) to the count matrix prior to cumulative sum scaling (CSS) [4], DESeq [30], and $\operatorname{logUQ}$ [28] transformation as a way to avoid $\log (0)$ is not ideal, because clustering results have been shown to be very sensitive to the choice of pseudocount, due to the nonlinear nature of the log transform [36, 37]. This underscores the need for a better solution to the zero problem so that logratio approaches inspired by Aitchison can be used [5] and is especially critical because matrices of microbial counts routinely contain zero values for an overwhelming majority of entries [4]. However, recent work has been done on Bayesian methods for zero estimation, with promising results [38, 60]. Furthermore, DESeq and edgeR-trimmed mean by $M$ values (TMM) make the assumptions that most microbes are not differentially abundant, and of those that are, there is an approximately balanced amount of increased/decreased abundance [28]; these assumptions are likely not appropriate for highly diverse microbial environments.

The simulations of Fig. 2 and Additional file 1: Figure S1 are relatively simple-the median library size of the two groups is approximately the same and there is no preferential sequencing. Hence, techniques like no normalization, or sample proportions, do well particularly in weighted UniFrac. It could be argued that if there were preferential sequencing in this simulation, CSS normalization would exhibit superior performance for weighted metrics [4, 36, 37]. It is regrettably beyond the scope of this paper to prove the "correct" normalization technique, but we further examine the unweighted measures.

We next applied the normalization techniques to several datasets from the literature to assess performance in light of the additional complexity inherent to real-world data. To perform an initial, detailed comparison of normalization methods, we selected the data set from Gevers et al. [10]. The data was the largest pediatric Crohn's disease cohort at the time of publication. The rarefied data was rarefied to 3000 sequences/sample, for all other normalization method samples with fewer than 3000 sequences/sample were removed from the raw data.

Using the data set from Gevers et al. [10], we observed substantial biases/confounding of results due to sequencing depth in PERMANOVA [61], partially because of low biological effect size $\left(R^{2}\right.$ or sum of squares' group/total sum of squares) (Fig. 3). In the ordination of unweighted UniFrac distance by PCoA, all normalization methods except rarefying exhibited library size effects of similar $R^{2}$ magnitude as nonnormalized data (Fig. 3a). DESeq and edgeR found no difference in the samples using unweighted UniFrac, potentially because of pseudocount addition. Thus, particularly with presence/absence metrics and low effect sizes, proper normalization is critical.

PCoA plots using ecologically common metrics for all of the normalization techniques on a few key real datasets representing a gradient [62], distinct body sites [63], and time series [64] are shown in Additional files 2 and 3: Figures S2-S3. Most normalization methods group according to biology in these cases where there is strong biologic difference. However, some clustering according to depth persists. For example, in the "Moving Pictures of the Human Microbiome" dataset [64], there is secondary clustering by sequence depth within each of the four main clusters when normalization alternatives to rarefying are applied.

Thus, both simulations and real data suggest that rarefying remains a useful technique for sample normalization prior to ordination and clustering for presence/absence distance metrics that have historically been very useful (such as binary Jaccard and unweighted UniFrac [57] distances). Other methods, for weighted distance measures and when sequencing depth is not a confounding variable, are promising. A more thorough study of the effects of compositional data on beta-diversity analysis is needed, as well as better tests for risk of incorrect results due to compositional data; we briefly investigate this topic in the next section.

\section{Differential abundance testing}

Many statistical methods have been proposed in the literature to compare the (relative) taxon abundance between two groups (e.g., case vs. control). Some statistical methods developed specifically for RNA-Seq data, such as DESeq [30], DESeq2 [42], edgeR [27, 44], and Voom [43] (Table 2), have been proposed for use on microbiome data [18] (note that because we found DESeq to perform similarly to DESeq2, except for very slightly lower sensitivity and false discovery rate (FDR), the former is not explicitly included in our results). On the other hand, metagenomeSeq $[4,65]$ and analysis of composition of microbiomes (ANCOM) [7] were developed specifically for microbial datasets, which usually contain many more zeros than RNA-Seq data. Except for ANCOM, all these approaches incorporate more sensitive statistical tests than the standard nonparametric tests such as the Wilcoxon rank-sum test, and they make some distributional assumptions. Therefore, they may have greater power to detect differentially abundant rare OTUs. Although ANCOM uses the Mann-Whitney test to avoid any distributional assumptions, which are not always easy to verify, its power can be improved by replacing the Mann-Whitney test by a parametric test while making some distributional assumptions.

Previous work in this area concluded that the newer differential abundance testing methods are worthwhile, and that the traditional practice of rarefying causes a high 


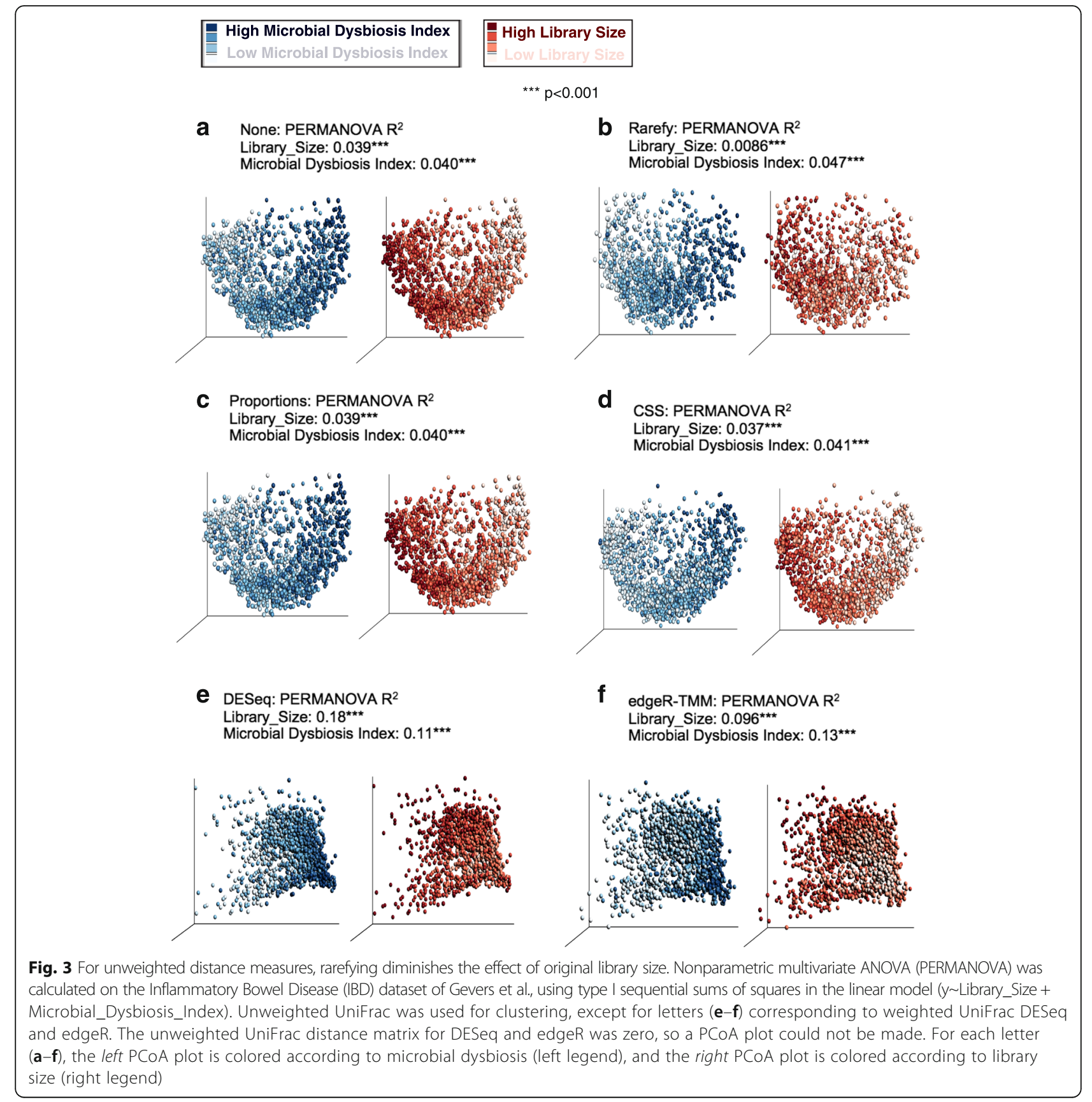

rate of false discoveries [18]. However, the latter conclusion was due to an artifact of the simulation (see the "Methods" section; Additional file 4: Figure S4a, Additional file 5: Statistical Supplement A and B). Instead, we found that rarefying itself does not cause a high rate of false discoveries, but rather leads to false negatives (lower sensitivity) driven by the discarding of data and the nonparametric nature of the Wilcoxon rank-sum (Figs. 4 and 5). The severity of the power decrease caused by rarifying depends upon how much data has been thrown away and how many samples were collected. This problem has been known for a long time, leading to the general guideline to rarefy to the highest depth possible [66]. If samples are discarded, justification should be explained.

We recognize that the multinomial and the Dirichletmultinomial (DM) distributions are not necessarily appropriate for the microbial taxon counts because under such probability distributions, every pair of taxa are negatively correlated, whereas as discussed in Mosimann [39] and Mandal et al. [8], not every pair of OTUs is in fact likely to be negatively correlated. However, because multinomial and DM distributions have been used by several authors 
Table 2 Differential abundance methods investigated in this study

\begin{tabular}{|c|c|}
\hline Method & Description \\
\hline Wilcoxon rank-sum test & $\begin{array}{l}\text { Also called the Mann-Whitney } U \text { test. A non-parametric rank test, which is used on the un-normalized } \\
(\text { "None"), proportion normalized, and rarefied matrices }\end{array}$ \\
\hline DESeq2 & $\begin{array}{l}\text { nbinomWald Test-The negative binomial GLM is used to obtain maximum likelihood estimates for an OTU's } \\
\text { log-fold change between two conditions. Then Bayesian shrinkage, using a zero-centered normal distribution } \\
\text { as a prior, is used to shrink the log-fold change towards zero for those OTUs of lower mean count and/or with } \\
\text { higher dispersion in their count distribution. These shrunken long fold changes are then used with the Wald } \\
\text { test for significance }\end{array}$ \\
\hline edgeR & $\begin{array}{l}\text { exact Test-The same normalization method (in } R \text {, method = RLE) as DESeq is utilized, and for differential } \\
\text { abundance testing also assumes the NB model. The main difference is in the estimation of the dispersion, } \\
\text { or variance, term. DESeq estimates a higher variance than edgeR, making it more conservative in } \\
\text { calling differentially expressed OTUs }\end{array}$ \\
\hline Voom & $\begin{array}{l}\text { Variance modeling at the observational level_library sizes are scaled using the edgeR log counts per } \\
\text { million (cpm) normalization factors. Then LOWESS (locally weighted regression) is applied to incorporate } \\
\text { the mean-variance trend into precision weights for each OTU }\end{array}$ \\
\hline \multirow[t]{2}{*}{ metagenomeSeq } & $\begin{array}{l}\text { fitZIG_-a zero-inflated Gaussian (ZIG) where the count distribution is modeled as a mixture of two distributions: } \\
\text { a point mass at zero and a normal distribution. Since OTUs are usually sparse, the zero counts are modeled with } \\
\text { the former, and the rest of the log transformed counts are modeled as the latter distribution. The parameters for } \\
\text { the mixture model are estimated with an expectation-maximization algorithm, which is coupled with } \\
\text { a moderated } t \text { statistic }\end{array}$ \\
\hline & $\begin{array}{l}\text { fitFeatureModel_-a feature-specific zero-inflated lognormal model with empirical Bayes shrinkage of } \\
\text { parameter estimates }\end{array}$ \\
\hline ANCOM & $\begin{array}{l}\text { Analysis of composition of microbiomes-compares the log ratio of the abundance of each taxon to the } \\
\text { abundance of all the remaining taxa one at a time. The Mann-Whitney } U \text { is then calculated on each log ratio }\end{array}$ \\
\hline
\end{tabular}

$[50,67,68]$, we include those distributions in our simulation study for purely comparative purposes. Additionally, we simulate data using the gamma-Poisson [7] (Figs. 4 and 5 and Additional files 6 and 7: Figures S5-S6). As expected, sensitivity increased with library size, but much more so for higher sample sizes, for all methods. Again as expected, for the nonparametric methods and small sample sizes, sensitivity was lower compared to parametric methods. For the parametric methods, in particular fitZIG and edgeR, the underlying data distribution changed results dramatically (Fig. 5 and Additional file 7: Figure S6). This is likely due to each model's distributional assumptions, e.g., the gamma-Poisson distribution is a closer relative to the negative binomial assumption of edgeR and DESeq; therefore, FDR is lower for the gamma-Poisson vs. the Dirichlet-multinomial. FitZIG was the only method where rarefying increased the FDR, because model parameters require original library size. Also, the 0.05 FDR thresholds were exceeded for techniques like DESeq2 and edgeR with more numbers of samples per group, possibly due to the increased degrees of freedom and decreased shrinkage of dispersion estimates.

One of the objectives of a microbiome study is to compare the abundance of taxa in the ecosystem of two or more groups using the observed taxa abundance in specimens drawn from the ecosystem. As noted in Mandal et al. (2015) [7] and in the Additional file 5: Statistical Supplement $\mathrm{C}$, even though the average taxa abundance of a taxon is the same in two ecosystems, it is not necessary that their mean relative abundances are same. Thus, drawing inferences regarding the mean taxon abundance between ecosystems using the specimen level data is a challenging problem. We performed a simulation study to evaluate the performance of various methods in terms of the false discovery rate and power when testing hypotheses regarding mean taxon abundance between ecosystems using the specimen level data. In simulations where the abundances of $10 \%$ of the OTUs increased in one group, all but ANCOM [7] had a highly inflated average FDR, in some cases exceeding 40\% (Fig. 6). False discovery for all methods increases when the fold change increases, because a larger constant applied to one OTU's counts impacts the relative abundance of other OTUs. Proportion normalization is known to have high FDR when faced with compositional data [6]. For DESeq/DESeq2, poor performance may be due to the model's assumption that differentially abundant OTUs are not a large portion of the population [29] or the model's overdispersion estimates [4]. Because most researchers want to infer ecosystem taxon relative abundances from sampling, this indicates a large previously unsolved problem in differential abundance testing [6].

We also investigated the performance of the techniques on real null data, in which there should be no "true positives," since samples from the same biological group were randomly divided into two groups ("Methods" section). 


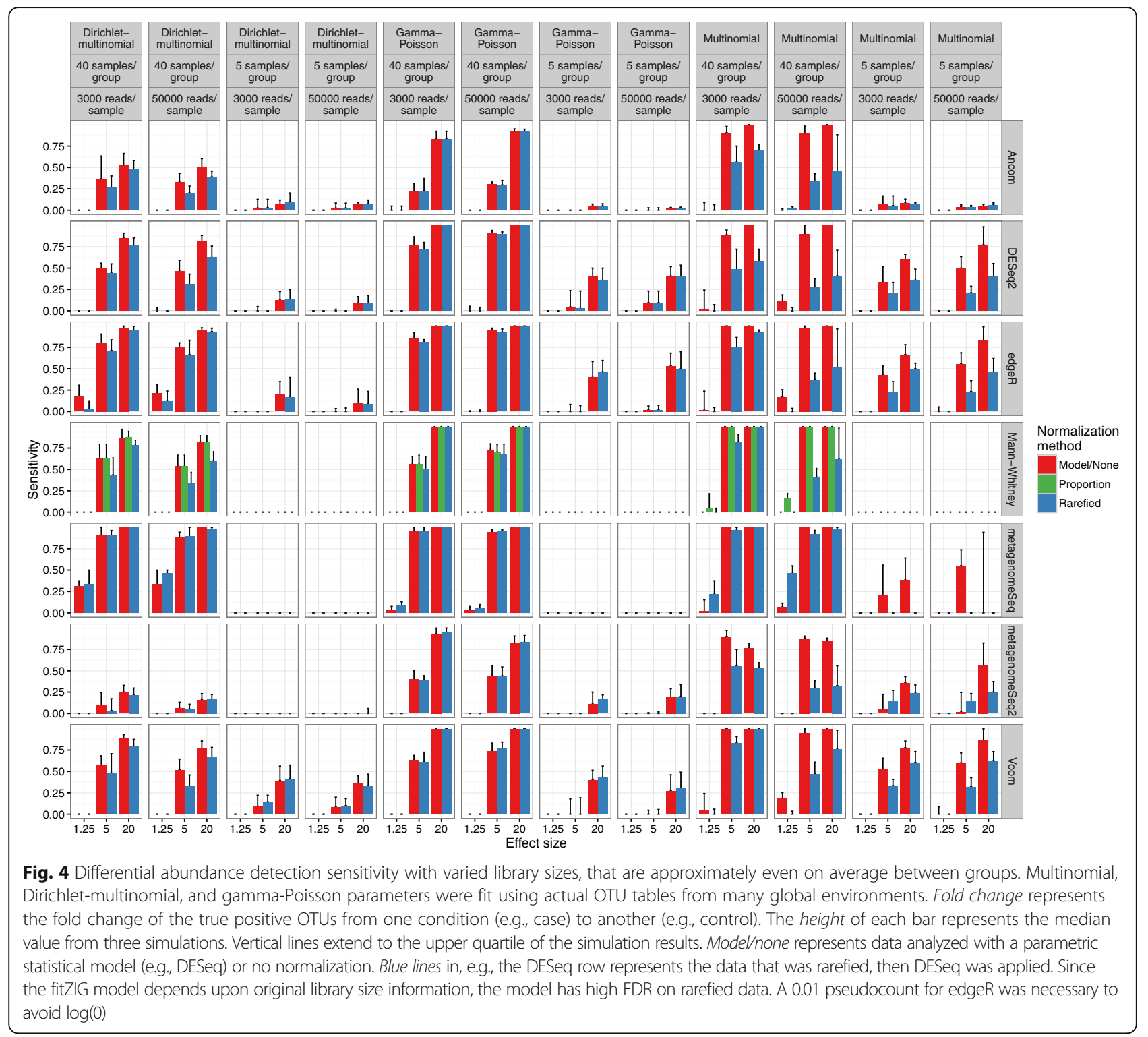

Most methods, except fitZIG, correctly predict no or very few false positives and are more conservative with decreasing sample size. However, for uneven library sizes and with 20-100 samples per group (Fig. 7a-b), the type 1 error rate of many methods increases beyond the nominal threshold. This was especially so for the no normalization and proportion normalization approaches. We did not observe increased type 1 error with ANCOM. The lack of increased type I error with rarefied data could simply be due to the loss of power resulting from rarefied data [18]. Additionally, manually adding a pseudocount (e.g., one) to the data matrix increases the type 1 error rate beyond the nominal threshold for uneven library sizes (Additional file 8: Figure S7a-b). Instead, one may consider standard zero imputation methods [69]. Interestingly, in the case of very small systematic biases (median effect size $<1$ ) as present in the raw data of Fig. 7c, the $t$ test on proportion-normalized data outperforms the nonparametric Wilcoxon rank-sum test in Fig. 7. This suggests that in the case of very small systematic biases, rankbased non-parametric tests (except fitZIG) could actually underperform parametric tests, as they do not take into account effect sizes. However, more investigation is necessary.

While the no normalization or proportion approaches control the FDR in cases where the average library size is approximately the same between the two groups (Figs. 4 and 5), they do not when one library is $10 \times$ larger than the other (Figs. 3 and 7). Therefore, we reiterate that neither the no normalization nor the sample proportion approach should be used for most statistical analyses. To demonstrate this, we suggest the theoretical 


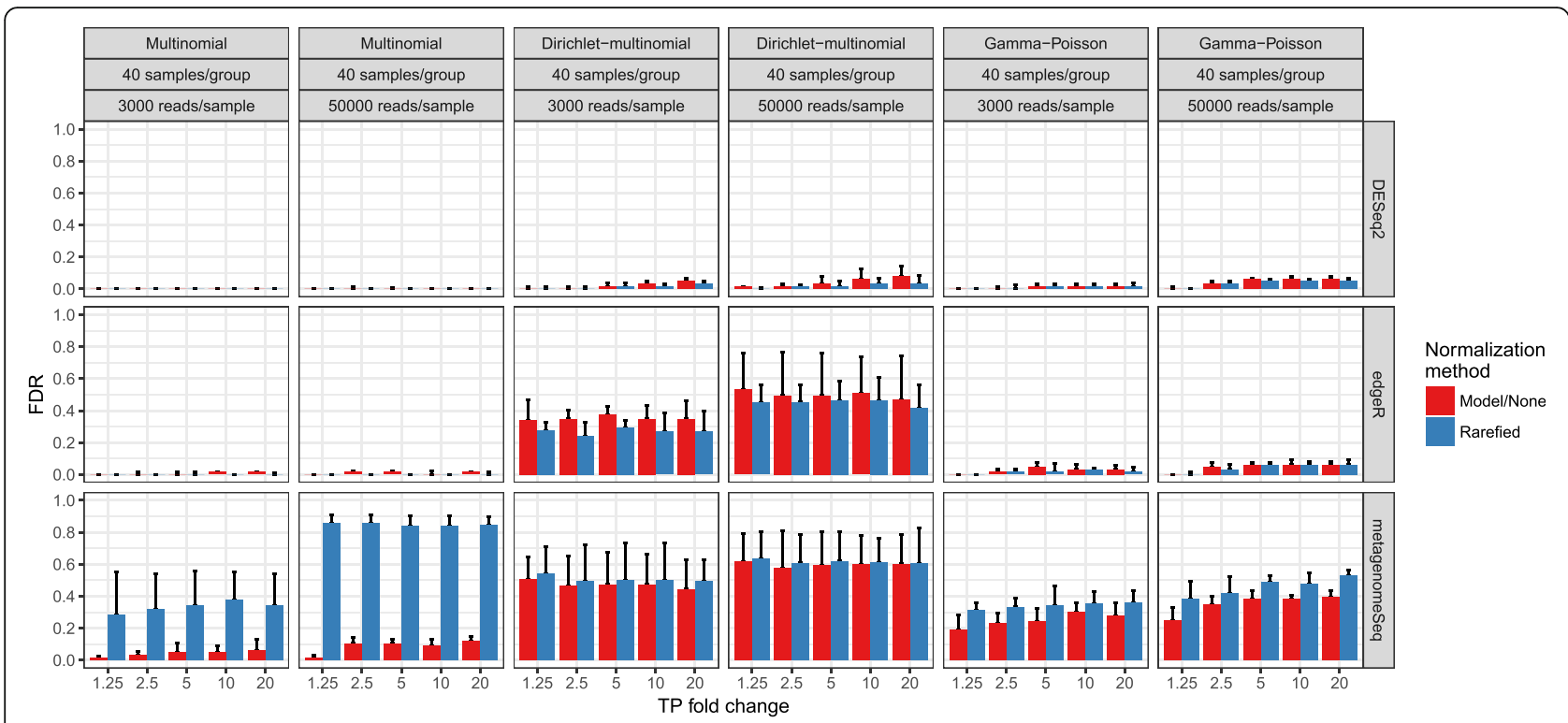

Fig. 5 Differential abundance detection false discovery rate with varied library sizes that are approximately even on average between groups. For simplicity, only those methods where the FDR exceeds or is close to 0.05 are shown. Full methods are in Additional file 7: Figure S6. Labels are the same as in Fig. 4

example of a data matrix with half the samples derived from diseased patients and half from healthy patients. If the samples from the healthy patients have a $10 \times$ larger library size, OTUs of all mean abundance levels will be found to be differentially abundant simply because they may have $10 \times$ the number of counts in the healthy patient samples (such systematic bias can happen if, for example, healthy vs. diseased patients are sequenced on separate sequencing runs or are being compared in a meta-analysis). The same warning applies for proportions, especially for rare OTUs that could be deemed differentially abundant because the rare OTUs may not be detected (zero values) in low library size samples, but are non-zero in high library size samples.

Using real data, we further tested the techniques shown to be most promising in the simulations: DESeq2 [42], metagenomeSeq $[4,65]$, rarefying with the Mann-Whitney $\mathrm{U}$ test, and ANCOM [7]. Ranges of dataset sizes were analyzed for environments that likely contain differentially abundant OTUs, as evidenced by the previously published PCoA plots and significance tests (Fig. 8). Six human skin and eight soil samples from Caporaso et al. [70], 28 samples in each of the lean vs. obese groups from Piombino et al. [71], and 500 samples in each of the tongue vs. left palm groups from Caporaso et al. [64] were tested. Although we do not necessarily know which OTUs are true positives in these actual data, it is of interest to investigate how the most promising techniques compare to each other. edgeR, which is known to underestimate dispersion, resulting in a high FDR $[4,42,45,53]$, predicts an extremely large number of significantly differentially abundant OTUs relative to other methods, especially for studies with small sample sizes (Fig. 8a). Additionally, in Fig. 8a, DESeq and edgeR predict well over half the OTUs to be differentially abundant, a violation of the associated normalization assumptions of constant abundance of a majority of species. While the disagreement in significantly differentially abundant OTU predictions decreases with increased library size, there is concern that simulations, no matter how carefully constructed, cannot mimic the complexity of real microbiome data.

\section{Conclusions}

We confirm that recently developed more complex techniques for normalization and differential abundance testing hold potential. Of methods for normalizing microbial data for ordination analysis, we found that DESeq normalization [30, 42], which was developed for RNA-Seq data and makes use of a $\log$-like transformation, does not work well with ecologically useful metrics, except weighted UniFrac [58]. DESeq normalization requires more development for general use on microbiome data. With techniques other than rarefying, library size is a frequent confounding factor that obscures biologically meaningful results. This is especially true with very low library sizes (under approximately 1000 sequences per sample) or if presence/absence metrics like unweighted UniFrac are used [57]. Additionally, many microbial environments are extremely variable in microbial composition, which would violate DESeq and edgeR-TMM normalization assumptions of a constant abundance of a majority of species and of a balance of increased/decreased abundance for those species that do change. Therefore, 


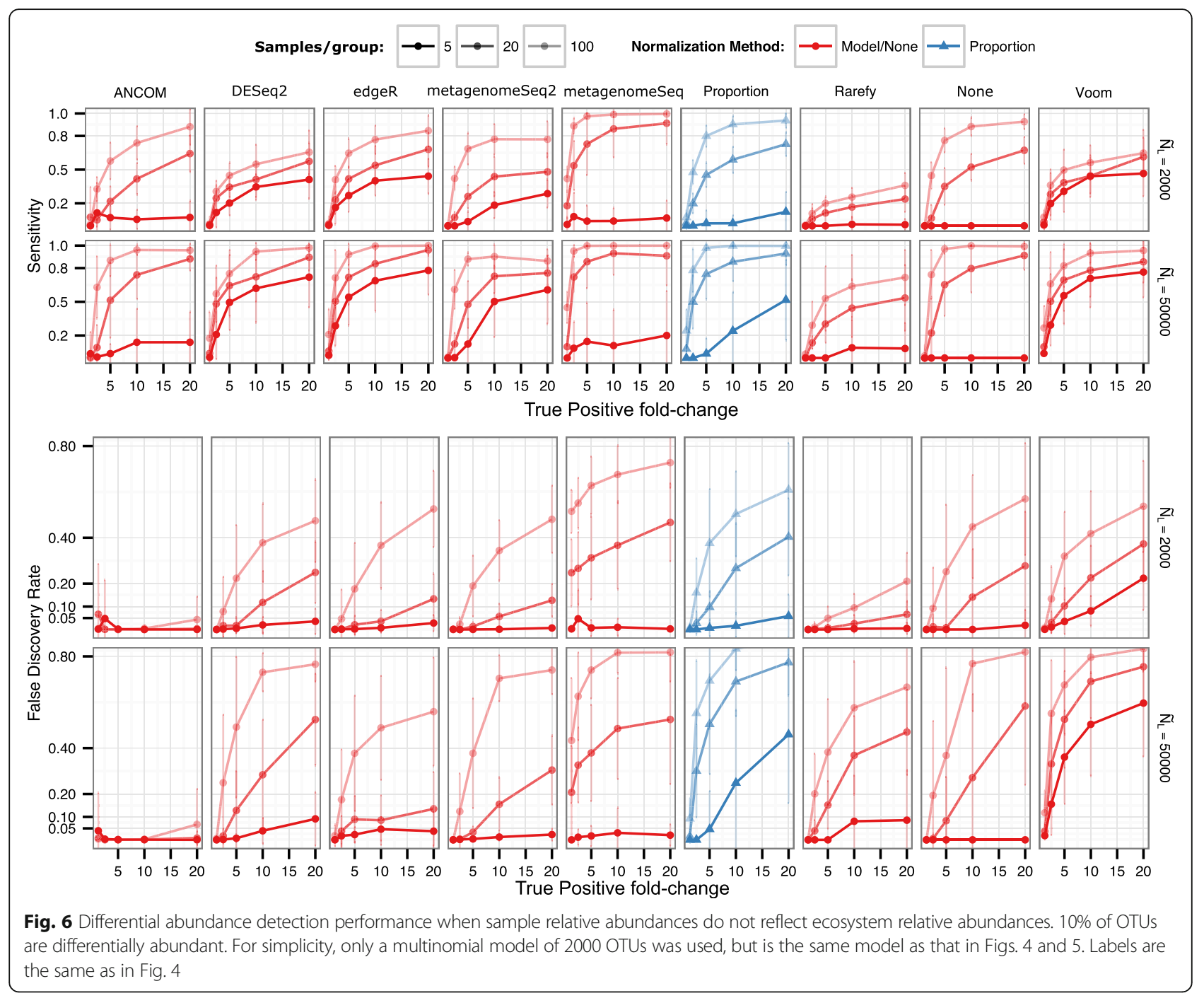

rarefying is still a useful normalization technique: rarefying can more effectively mitigate the artifact of sample library size than other normalization techniques and results in a higher PERMANOVA $R^{2}$ for the studied biological effect, especially for small ( $<1000$ sequences per sample) and very uneven ( $>\sim 10 \times$ on average) library sizes between groups. The approaches of no normalization and sample proportion are prone to generation of artifactual clusters based on sequencing depth in beta diversity analysis. Therefore, researchers should proceed with caution and check for these effects in ordination results if the count data was not rarefied. In PERMANOVA tests, we recommend that a term for library size is included if the data set was not rarefied or otherwise normalized.

For differential abundance testing, we used both simulations and real data. Overall, we found that simulation results are very dependent upon simulation design and distribution, highlighting the need for gold standard datasets. We confirm that techniques based on GLMs with the negative binomial or log-ratios are promising. DESeq2 [42] was designed for, and provides, increased sensitivity on smaller datasets ( $<20$ samples per group); however, it tends towards a higher false discovery rate with larger and/or very uneven library sizes ( $>\sim 10 \times$ on average). The practice of manually adding a pseudocount to the matrix prior to DESeq2 transformation increases the FDR. This agrees with prior investigation finding RNA-Seq approaches unsuitable for microbiome data [60]. If the average library size for each group is approximately equal, then rarefying itself does not increase the false discovery rate. For groups with large $(\sim 10 \times)$ differences in the average library size between groups, rarefying helps decrease the false discovery rate. Prior to analysis, researchers should assess the difference in average library size between groups. If large variability in library sizes across samples is observed, then rarefying is useful as a method of normalization. ANCOM [7] maintains a low FDR for all sample sizes and is the only method that is suitable for making inferences regarding the taxon 


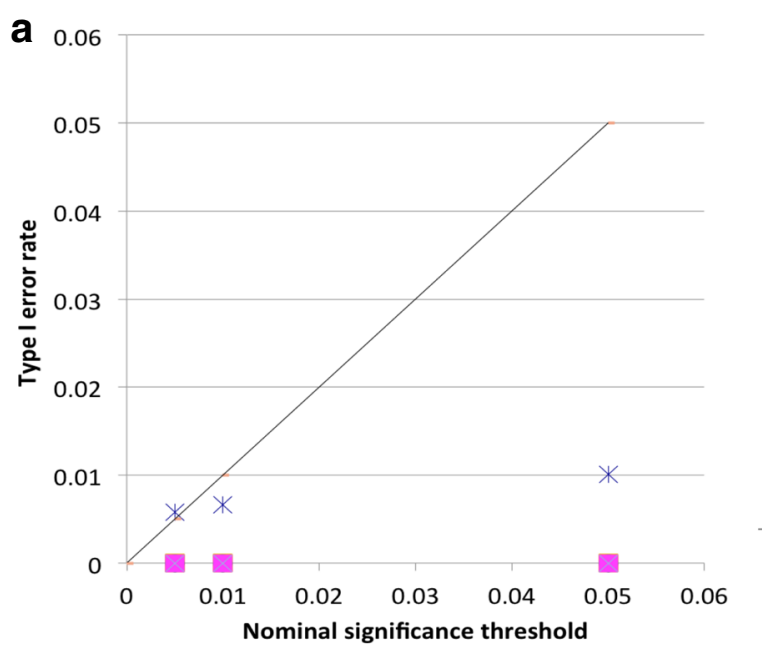

- None - wrs

- Proportion - wrs

$\times$ Proportion - ttest

- Rarefy - wrs

- Rarefy - ANCOM

- DESeq2

$*$ fitZIG

- fitFeatureModel

edgeR

- Linear (45-degree)

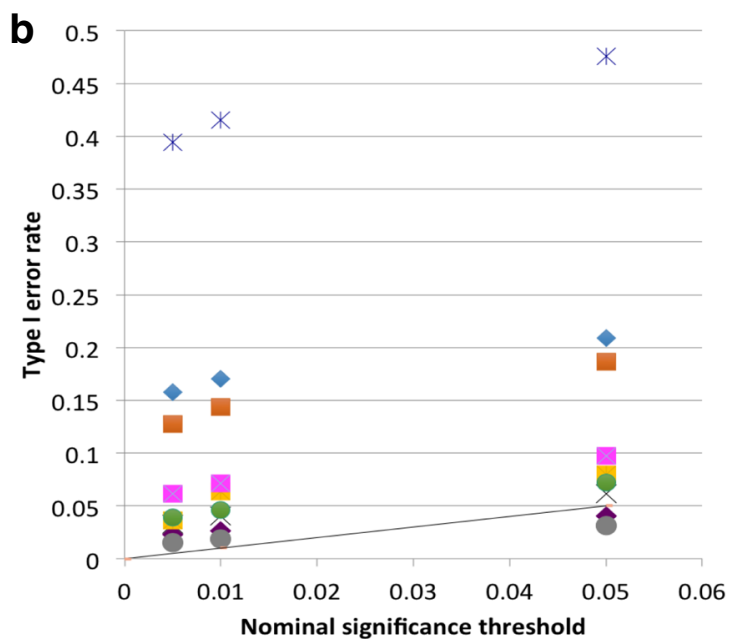

- None - wrs

- Proportion - wrs

$\times$ Proportion - ttest

- Rarefy - wrs

- Rarefy - ANCOM

DESeq2

* fitZIG

fitFeatureModel

adgeR

Linear (45-degree)

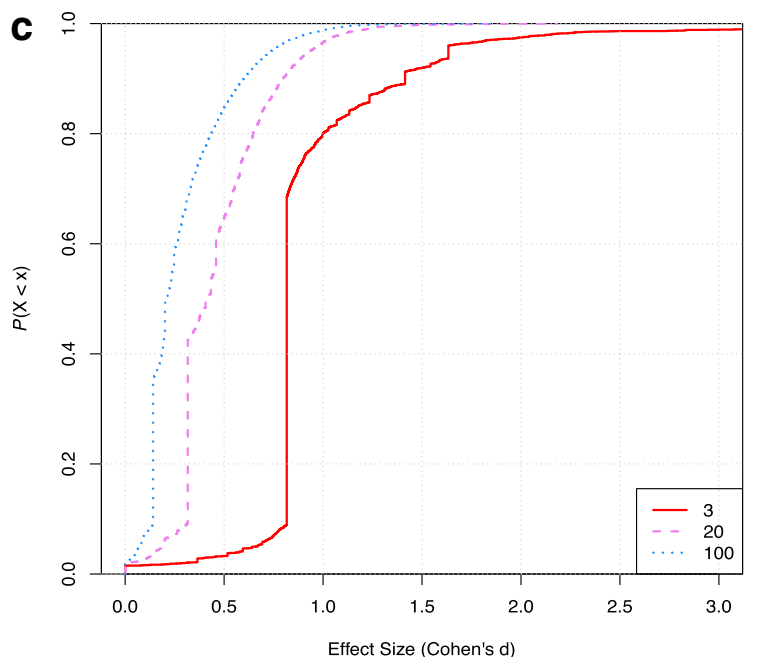

Fig. 7 False discovery rate increases when methods are challenged with very uneven library sizes. Real data from one body site was randomly divided into two groups, creating a situation in which there should be no true positives. a Uneven library sizes, 3 samples per group. b Uneven library sizes, 100 samples per group. For uneven library sizes, the group means differed by 10x (e.g., 40,000 sequences per sample vs. 4000 sequences per sample). The 45-degree line shows where the nominal FDR should equal the observed FDR. c Cumulative distribution functions of the effect sizes for 3, 20, and 100 samples per group presented in $\mathbf{a}$ and $\mathbf{b}$. Voom was excluded because it was found to have a higher type I error rate than fitZIG 

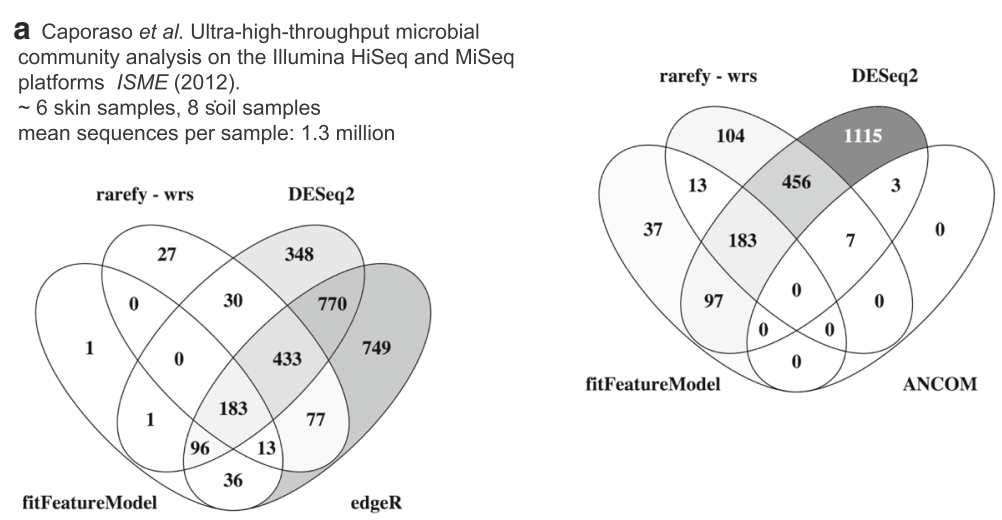

b Piombino et al. Saliva from Obese Individuals Suppresses the Release of Aroma Compounds from Wine. PLoS One (2014).

$\sim 28$ samples per category (lean vs. obese) mean sequences per sample: 75,580

C Caporaso et al. Moving Pictures of the Human Microbiome. Genome Biology (2011) $\sim 500$ samples per category (tongue vs. left palm) mean sequences per sample: 25,600
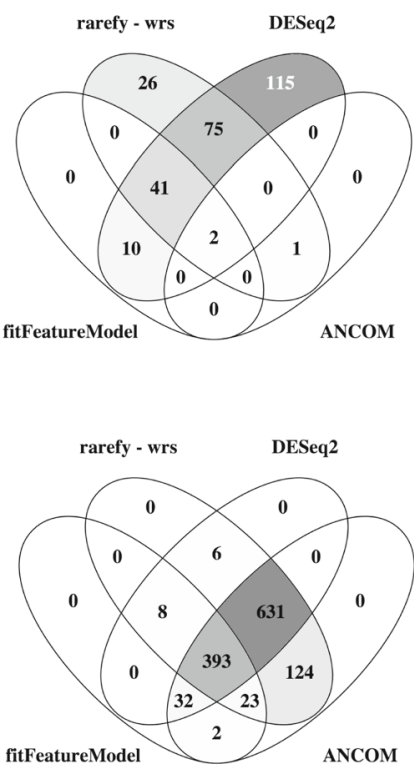

Fig. 8 On real datasets, methods disagree especially for few samples per group. FDR $p<0.05$. Darker colors indicate a larger proportion of OTUs discovered by a technique or combination of techniques

abundance (as well as the relative abundance) in the ecosystem using the abundance data from specimens. With ANCOM, sensitivity is decreased on small datasets $(<20$ samples per group), partially due to its use of the Mann-Whitney test. ANCOM with more sensitive statistical tests needs to be investigated.

Thanks to McMurdie and Holmes' previous work in this area [18], we recognize the potential of these newer techniques and have incorporated DESeq2 [42] and metagenomeSeq $[4,65]$ normalization and differential abundance testing into QIIME version 1.9.0 [24], along with the traditional rarefying and non-parametric testing techniques. ANCOM differential abundance testing is included in scikitbio (scikit-bio.org) and will be part of QIIME version 2.0.

\section{Methods}

\section{Normalization}

The basic test of how well broad differences in microbial sample composition are detected, as assessed by clustering analysis, was conducted in "simulation A" from McMurdie and Holmes [18]. Briefly, the "ocean" and "feces" microbiomes (the microbial data from ocean and human feces samples, respectively) from the "Global Patterns" dataset [72] were used as templates, modeled with a multinomial, and taken to represent distinct classes of microbial community because they have few OTUs in common. These two classes were mixed in eight defined proportions (the "effect size") in independent simulations in order to generate simulated samples of varying clustering difficulty. Samples were generated in sets of 40 , as in McMurdie and Holmes [18]. We also tested smaller and larger sample sizes but saw little difference in downstream results. Additional sets of 40 samples were simulated for varying library sizes $(1000,2000$, 5000 , and 10,000 sequences per sample). These simulated samples, done in triplicate for each combination of parameters, were then used to assess normalization 
methods by the proportion of samples correctly classified into the two clusters by the partitioning around medioids (PAM) algorithm [73, 74].

McMurdie and Holmes [18] evaluated clustering accuracy with five normalization methods (none, proportion, rarefying with replacement as in the multinomial model [75], DESeqVS [30], and UQ- $\operatorname{logFC}$ (in the edgeR package) [27]) and six beta-diversity metrics (Euclidean, Bray-Curtis [59], PoissonDist [76], top-MSD [27], unweighed UniFrac [57], and weighted UniFrac [58]). We modified the normalization methods to those in Table 1 (none, proportion, rarefying without replacement as in the hypergeometric model [75], CSS [4], logUQ [28], DESeqVS [30], and edgeR-TMM [27]) and the beta diversity metrics to those in Fig. 2 and Additional file 1: Figure S1 (binary Jaccard, Bray-Curtis [59], Euclidean, unweighed UniFrac [57], and weighted UniFrac [58]), thus including more recent normalization methods [4, 28 ] and only those beta diversity metrics that are most common in the literature. We amended the rarefying method to the hypergeometric model [75], which is much more common in microbiome studies [23, 24]. Negative values in the DESeq normalized values [30] were set to zero as in McMurdie and Holmes [18], and a pseudocount of one was added to the count tables [18]. McMurdie and Holmes [18] penalized the rarefying technique for dropping the lowest fifteenth percentile of sample library sizes in their simulations by counting the dropped samples as "incorrectly clustered." Because the 15 th percentile was used to set rarefaction depth, this capped clustering accuracy at $85 \%$. We instead quantified cluster accuracy among samples that were clustered following normalization to exclude this rarefying penalty (Fig. 2). Conversely, it has since been confirmed that low-depth samples contain a higher proportion of contaminants (rRNA not from the intended sample) $[55,56]$. Because the higher depth samples that rarefying keeps may be higher quality and therefore give rarefying an unfair advantage, Additional file 1: Figure S1 compares clustering accuracy for all the techniques based on the same set of samples remaining in the rarefied dataset.

On the real datasets, nonparametric multivariate ANOVA (PERMANOVA) [77] was calculated by fitting a type I sequential sums of squares in the linear model (y Library_Size + Biological_Effect). Thus, we control for library size differences before assessing the effects on the studied biological effect. All data was retrieved from QIITA (https://qiita.ucsd.edu/).

\section{Differential abundance testing \\ Multinomial distribution}

The simulation test for how well truly differentially abundant OTUs are recognized by various parametric and nonparametric tests was conducted as in "simulation B" in McMurdie and Holmes [18], with a few changes. The basic data generation model remained the same, but the creation of true positive OTUs was either made symmetrical through duplication or moved to a different step, so that the OTU environmental abundances matched their relative abundances. The "Global Patterns" [72] dataset was again used, because it was one of the first studies to apply high-throughput sequencing to a broad range of environments, which includes nine environment types from "ocean" to "soil"; all simulations were evaluated for all environments. Additionally, we verified the results on the "lean" and "obese" microbiomes from a different study [71]. As in McMurdie and Holmes, correction for multiple testing was performed using the Benjamini \& Hochberg [78] FDR threshold of 0.05 .

A simple overview of the two methods used for simulating differential abundance is presented in Additional file 4: Figure S4a. In McMurdie and Holmes' [18] "original" simulation (second row), the distribution of counts from one environment (e.g., ocean) was modeled off of a multinomial template (first row) for two similar groups (ocean_1 and ocean_2), ensuring a baseline of all "true negative" OTUs. Following the artificial inflation of specific OTUs in the ocean_1 samples to create true positives, fold-change estimates for every other OTU are affected. Thus, although in terms of abundances, their set-up allows for some true positives and true negatives, in terms of relative abundances, by their sampling scheme, some taxa are true positives. Thus, true negatives are possible true positives in terms of relative abundances. To ensure that not all taxa are true positives in terms of relative abundances, we created pairs of differentially abundant OTUs in both the ocean_1 and ocean_2 samples (third row), and thus created a new "balanced" simulation where the same taxa are differentially abundant as well as differentially relative abundant. Details are in Additional file 5: Statistical Supplement $\mathrm{A}$ and $\mathrm{B}$.

However, in general, as noted in Additional file 5: Statistical Supplement $C$, equality of taxa abundance between two environments does not translate to equality of the relative abundance of taxa between two environments. In terms of statistical tests, depending upon what parameter is being tested, this can result in inflated false discovery rates. To illustrate this phenomenon, we conducted a simulation study mimicking Additional file 4: Figure S4b, with results in Fig. 6, where two environments had differentially abundant taxa. Samples were generated from such environments according to a multinomial distribution and these specimen level data were used to compare the taxa abundance in the two environments. 
Besides the above procedural changes to the McMurdie and Holmes [18] simulation, we also modified the rarefying technique from sampling with replacement (multinomial) to sampling without replacement (hypergeometric-as in the previous normalization simulations) [75]. The testing technique was modified from a two-sided Welch $t$ test to the nonparametric Mann-Whitney test, which is widely used and more appropriate because the OTU distributions in microbiome data usually deviate from normality. The techniques used (Table 2) differ only by the addition of another metagenomeSeq method, fitFeatureModel [65], another RNA-Seq method, Voom [43], and ANCOM [8]. This new simulation code, for which all intermediate files and dependencies are easily available, can be found in the supplemental $R$ files (Additional file 9 and 10).

\section{Dirichlet-multinomial distribution}

This simulation was exactly the same as the above "multinomial" simulation, except that the Dirichlet-multinomial distribution was used instead of the multinomial distribution to model the nine environments found in the Global Patterns [72] dataset. Dirichlet-multinomial was a better fit for the Global Patterns data, as determined through the "HMP" package [67] and the $C(\alpha)$-optimal test-statistics [79]. Dirichlet-multinomial parameters were calculated through the method of moments estimators. As a check, we ensured that the Dirichlet-multinomial results converged to the multinomial results with large gamma.

\section{Gamma-Poisson distribution}

This simulation was exactly the same as the above multinomial simulation, except that the gamma-Poisson distribution was used instead of the multinomial distribution to model the nine environments found in the Global Patterns [72] dataset. The means and the variances of the OTUs across samples for each of the environments were used to estimate the lambdas of the gamma-Poisson distribution. As a check, we ensured that the gamma-Poisson results converged to the multinomial results with large shape parameter.

\section{Real null data}

For the experimental, null data test, we selected random samples from within one body site ("left hand") of Caporaso et al. [64]. We then randomly divided the samples into two groups, each having 3,20 , and 100 samples, and applied the differential abundance methods. For the case where the library sizes between the two groups differed by $\sim 10 \times$, we selected some of the lowest and highest library size samples within the left hand group.

\section{ANCOM}

The ANCOM procedure compares the relative abundance of a taxon between two ecosystems by computing Aitchison's [5] log-ratio of abundance of each taxon relative to the abundance of all remaining taxa one at a time. Thus, if there are " $\mathrm{m}$ " taxa, then for each taxon it performs " $\mathrm{m}-1$ " tests and the significance of each test is determined using the Benjamini-Hochberg procedure that controls for FDR at 0.05 . For each taxon, ANCOM counts the number of tests among the m-1 tests that are rejected. Thus for each taxon, ANCOM obtains a count random variable $W$ that represents the number of nulls among the m-1 tests that are rejected. ANCOM determines the final significance of a taxon by using the empirical distribution of $W$. To deal with zero counts, we use an arbitrary pseudo count value of 0.001. For a more detailed description of ANCOM, we refer the reader to Mandal et al. [7].

\section{Additional files}

Additional file 1: Figure S1. Comparison of common distance metrics and normalization methods when low-coverage samples are excluded. The right axis represents the median library size $\left(\mathrm{N}_{1}\right)$, while the $\mathrm{x}$-axis effect size is the multinomial mixing proportions of the two classes of samples, ocean and feces. For rarefying, samples below the 15th percentile of library size were dropped from the analysis. See caption for Fig. 2 for further details. (PDF 432 kb)

Additional file 2: Figure S2. All normalization techniques on key microbiome datasets, Bray Curtis distance. Rows of panels show (from top to bottom) data from 88 soils [62], body sites [63], and moving pictures [64]. 88 soils are colored according to a color gradient from low to high $\mathrm{pH}$. The Costello et al. body sites' dataset is colored according to body site feces (b/ue) and oral cavity (purple); the rest of the colors are external auditory canal, hair, nostril, skin, and urine. Moving pictures dataset: left and right palm (red/blue), tongue (green), and feces (orange). It is important to note that all the samples in these datasets are approximately the same depth, and there are very strong driving gradients. (PNG $1357 \mathrm{~kb}$ )

Additional file 3: Figure S3. All normalization techniques on key microbiome datasets, unweighted UniFrac distance. See Figure S3 caption for details. (PNG 1368 kb)

Additional file 4: Figure S4. Simple example of the reasoning behind differential abundance simulations. a In actual OTU tables generated from sequencing data, the counts (left column) are already compositional and therefore only relative. Application of a constant multiplier to the original multinomial template to create fold-change differences disturbs the distinction between true positive (TP) and true negative (TN) OTUs in the original simulation, but not the balanced simulation. $\mathbf{b}$ Creation of compositional data from the multinomial template. The sum constraint that occurs when a researcher samples from the environment can cause, e.g., unchanged OTUs to appear changed in an OTU table. (PDF 199 kb)

Additional file 5: Statistical supplement: statistical details of simulation studies. (DOCX 119 kb)

Additional file 6: Figure S5. Differential abundance detection sensitivity with varied library sizes that are approximately even on average between groups. Label the same as Fig. 4, but with more effect sizes. (PDF 44 kb)

Additional file 7: Figure S6. Differential abundance detection false discovery rate with varied library sizes that are approximately even on average between groups. An expanded Fig. 5. (PDF 40 kb)

Additional file 8: Figure S7. Pseudocount addition to avoid zero increases FDR. The same data as Fig. 7. a Uneven library sizes, FDR $p<0.05$. b Uneven library sizes, FDR $p<0.01$. Pseudo indicates a pseudocount of one was added to the matrix prior to analysis. (PDF $104 \mathrm{~kb}$ ) 
Additional file 9: Code adapted from McMurdie and Holmes [18] to include additional methods. This code is the basis for Figs. 4 and 5.

Additional file 10: Code adapted from McMurdie and Holmes [18] to include additional methods and compositional effects. This code is the basis for Figure 6.

\section{Acknowledgements}

We also thank Joseph N. Paulson, Joey McMurdie, and Jonathan Friedman for helpful conversations and Greg Caporaso and Jai Ram Rideout for coding advice.

\section{Funding}

SJW was funded by the National Human Genome Research Institute Grant No. 3 R01 HG004872-03S2 and the National Institute of Health Grant No. 5 U01 HG004866-04. Research of SDP was supported by the Intramural Research Program of the National Institute of Environmental Health Sciences, NIH (Z01 ES101744-04). AB's participation was partially funded by CTRI grant no. UL1TR001442

\section{Availability of data and materials}

The datasets supporting the conclusions of this article are included within the article (and its additional files). R version 3.1.0 [80] was used with Bioconductor [81] packages phyloseq version 1.10.0, HMP version 1.3.1, DESeq version 1.16.0, DESeq2 version 1.4.5, edgeR version 3.6.8, metagenomeSeq version 1.11.10, and Limma version 3.20.9. Also, we used Python-based QIIME version 1.9.0, with Emperor [82]

\section{Authors' contributions}

SJW, ZZX, AA, SDP, KB, AG, JRZ, and RK designed and conceived analyses. SJW wrote the QIIME scripts, and AGP and YVB helped integrate the scripts into QIIME. SJW wrote the initial manuscript, and all authors provided invaluable feedback and insights into analyses and the manuscript. All authors approved the final version of the manuscript.

\section{Competing interests}

The authors declare that they have no competing interests.

\section{Consent for publication}

\section{Not applicable.}

\section{Ethics approval and consent to participate}

This manuscript does not report on data collected from humans or animals.

\section{Author details}

${ }^{1}$ Department of Chemical and Biological Engineering, University of Colorado at Boulder, Boulder, CO 80309, USA. ${ }^{2}$ Departments of Pediatrics, University of California San Diego, 9500 Gilman Drive, MC 0763, La Jolla, CA 92093, USA. ${ }^{3}$ Biostatistics and Computational Biology Branch, NIEHS, NIH, Research Triangle Park Durham, NC, USA. ${ }^{4}$ Department of Microbiology, University of Pennsylvania, Philadelphia, PA 18014, USA. ${ }^{5}$ Department of Medicine, University of Colorado, Denver, CO 80204, USA. ${ }^{6}$ Department of Microbiology, Oregon State University, 226 Nash Hall, Corvallis, OR 97331, USA. ${ }^{7}$ Department of Computer Science \& Engineering, University of California San Diego, La Jolla, CA 92093, USA. ${ }^{8}$ Center for Computational Biology and Bioinformatics, Dept. of Medicine, University of California San Diego, La Jolla, CA 92093, USA. ${ }^{9}$ Center for Microbiome Innovation, University of California San Diego, La Jolla, CA 92093, USA.

Received: 9 October 2015 Accepted: 27 January 2017

Published online: 03 March 2017

\section{References}

1. Qin J, Li R, Raes J, Arumugam M, Burgdorf KS, Manichanh C, et al. A human gut microbial gene catalogue established by metagenomic sequencing. Nature. 2010;464(7285):59-65. doi:10.1038/nature08821.

2. Rodriguez RL, Konstantinidis KT. Estimating coverage in metagenomic data sets and why it matters. ISME J. 2014;8(11):2349-51. doi:10.1038/ismej.2014.76.

3. Lozupone C, Lladser ME, Knights D, Stombaugh J, Knight R. UniFrac: an effective distance metric for microbial community comparison. ISME J. 2011; 5(2):169-72. doi:10.1038/ismej.2010.133.
4. Paulson JN, Stine OC, Bravo HC, Pop M. Differential abundance analysis for microbial marker-gene surveys. Nat Methods. 2013;10(12):1200-2. doi:10.1038/nmeth.2658.

5. Aitchison J. The statistical analysis of compositional data. J Roy Stat Soc B Met. 1982;44(2):139-77.

6. Lovell D, Pawlowsky-Glahn V, Egozcue JJ, Marguerat S, Bahler J. Proportionality: a valid alternative to correlation for relative data. PLoS Comput Biol. 2015;11(3):e1004075. doi:10.1371/journal.pcbi.1004075.

7. Mandal S, Van Treuren W, White RA, Eggesbo M, Knight R, Peddada SD. Analysis of composition of microbiomes: a novel method for studying microbial composition. Microb Ecol Health Dis. 2015;26:27663. doi:10.3402/ mehd.v26.27663.

8. Friedman J, Alm EJ. Inferring correlation networks from genomic survey data PLoS Comput Biol. 2012;8(9):e1002687. doi:10.1371/journal.pcbi.1002687.

9. Gower JC. Some distance properties of latent root and vector methods used in multivariate analysis. Biometrika. 1966;53:325. doi:10.2307/2333639.

10. Gevers D, Kugathasan S, Denson LA, Vazquez-Baeza Y, Van Treuren W, Ren $B$, et al. The treatment-naive microbiome in new-onset Crohn's disease. Cell Host Microbe. 2014;15(3):382-92. doi:10.1016/j.chom.2014.02.005.

11. Pop M, Walker AW, Paulson J, Lindsay B, Antonio M, Hossain MA, et al. Diarrhea in young children from low-income countries leads to large-scale alterations in intestinal microbiota composition. Genome Biol. 2014;15(6): R76. doi:10.1186/gb-2014-15-6-r76.

12. Ley RE, Backhed F, Turnbaugh P, Lozupone CA, Knight RD, Gordon J. Obesity alters gut microbial ecology. Proc Natl Acad Sci U S A. 2005; 102(31):11070-5. doi:10.1073/pnas.0504978102.

13. Ridaura VK, Faith JJ, Rey FE, Cheng J, Duncan AE, Kau AL, et al. Gut microbiota from twins discordant for obesity modulate metabolism in mice. Science. 2013;341(6150):1241214. doi:10.1126/science.1241214.

14. Turnbaugh PJ, Hamady M, Yatsunenko T, Cantarel BL, Duncan A, Ley RE, et al. A core gut microbiome in obese and lean twins. Nature. 2009; 457(7228):480-4. doi:10.1038/nature07540.

15. Lozupone CA, Li M, Campbell TB, Flores SC, Linderman D, Gebert MJ, et al. Alterations in the gut microbiota associated with HIV-1 Infection. Cell Host Microbe. 2013;14(3):329-39. doi:10.1016/J.Chom.2013.08.006.

16. David LA, Maurice CF, Carmody RN, Gootenberg DB, Button JE, Wolfe BE, et al. Diet rapidly and reproducibly alters the human gut microbiome. Nature. 2014:505(7484):559-63. doi:10.1038/nature12820.

17. Lozupone CA, Stombaugh J, Gonzalez A, Ackermann G, Wendel D, VazquezBaeza Y, et al. Meta-analyses of studies of the human microbiota. Genome Res. 2013;23(10):1704-14. doi:10.1101/Gr.151803.112.

18. McMurdie PJ, Holmes S. Waste not, want not: why rarefying microbiome data is inadmissible. PLoS Comput Biol. 2014;10(4). doi:Artn E1003531. Doi 10.1371/Journal.Pcbi.1003531.

19. Gotelli NJ, Colwell RK. Quantifying biodiversity: procedures and pitfalls in the measurement and comparison of species richness. Ecol Lett. 2001;4(4): 379-91. doi:10.1046/J.1461-0248.2001.00230.X.

20. Brewer A, Williamson M. A new relationship for rarefaction. Biodivers Conserv. 1994;3(4):373-9. doi:10.1007/Bf00056509.

21. Horner-Devine MC, Lage M, Hughes JB, Bohannan BJ. A taxa-area relationship for bacteria. Nature. 2004;432(7018):750-3. doi:10.1038/ nature03073.

22. Jernvall J, Wright PC. Diversity components of impending primate extinctions. Proc Natl Acad Sci U S A. 1998;95(19):11279-83.

23. Schloss PD, Westcott SL, Ryabin T, Hall JR, Hartmann M, Hollister EB, et al. Introducing mothur: open-source, platform-independent, communitysupported software for describing and comparing microbial communities. Appl Environ Microbiol. 2009;75(23):7537-41. doi:10.1128/AEM.01541-09.

24. Caporaso JG, Kuczynski J, Stombaugh J, Bittinger K, Bushman FD, Costello EK, et al. QIIME allows analysis of high-throughput community sequencing data. Nat Methods. 2010;7(5):335-6. doi:10.1038/nmeth.f.303.

25. Jari Oksanen FGB, Roeland Kindt, Pierre Legendre, Peter R. Minchin, R. B. O'Hara, Gavin L. Simpson, Peter Solymos, M. Henry H. Stevens and Helene Wagner. Vegan: community ecology package. R package version 22-1. 2015.

26. McMurdie PJ, Holmes S. phyloseq: an R package for reproducible interactive analysis and graphics of microbiome census data. PloS one. 2013;8(4). doi: ARTN e61217 DOl 10.1371/journal.pone.0061217.

27. Robinson MD, McCarthy DJ, Smyth GK. edgeR: a Bioconductor package for differential expression analysis of digital gene expression data. Bioinformatics. 2010;26(1):139-40. doi:10.1093/Bioinformatics/Btp616. 
28. Bullard JH, Purdom E, Hansen KD, Dudoit S. Evaluation of statistical methods for normalization and differential expression in mRNA-Seq experiments. BMC Bioinformatics. 2010;11:94. doi:10.1186/1471-2105-11-94.

29. Dillies MA, Rau A, Aubert J, Hennequet-Antier C, Jeanmougin M, Servant N, et al. A comprehensive evaluation of normalization methods for Illumina high-throughput RNA sequencing data analysis. Brief Bioinform. 2013;14(6): 671-83. doi:10.1093/bib/bbs046.

30. Anders S, Huber W. Differential expression analysis for sequence count data. Genome Biol. 2010;11(10). doi:Artn R106. Doi 10.1186/Gb-2010-11-10-R106.

31. Agresti A, Hitchcock DB. Bayesian inference for categorical data analysis. Statistical Methods and Applications. 2005;14(3):297-330.

32. Buccianti A, Mateu-Figueras G, Pawlowsky-Glahn V. Compositional data analysis in the geosciences: from theory to practice. Geological Society special publication, vol no 264. London: The Geological Society; 2006.

33. Pearson K. Mathematical contributions to the theory of evolution: on a form of spurious correlation which may arise when indices are used in the measurements of organs. Proc Roy Soc. 1896;60:489-98.

34. Egozcue JJ, Pawlowsky-Glahn V, Mateu-Figueras G, Barcelo-Vidal C. Isometric logratio transformations for compositional data analysis. Math Geol. 2003;35(3):279-300. doi:10.1023/A:1023818214614.

35. Greenacre M. Measuring subcompositional incoherence. Math Geosci. 2011;43(6):681-93. doi:10.1007/S11004-011-9338-5.

36. Costea Pl, Zeller G, Sunagawa S, Bork P. A fair comparison. Nat Methods. 2014;11(4):359. doi:10.1038/nmeth.2897.

37. Paulson JN, Bravo HC, Pop M. Reply to: "a fair comparison". Nat Methods. 2014;11(4):359-60. doi:10.1038/nmeth.2898.

38. Martin-Fernandez JA, Hron K, Templ M, Filzmoser P, Palarea-Albaladejo J. Bayesian-multiplicative treatment of count zeros in compositional data sets. Stat Model. 2015;15(2):134-58.

39. Mosimann JE. On the compound multinomial distribution, the multivariate $\beta$-distribution, and correlations among proportions. Biometrika. 1962:49:65-82.

40. Wagner BD, Robertson CE, Harris JK. Application of two-part statistics for comparison of sequence variant counts. PLoS One. 2011;6(5):e20296. doi:10.1371/journal.pone.0020296.

41. Anders S, McCarthy DJ, Chen YS, Okoniewski M, Smyth GK, Huber W, et al. Count-based differential expression analysis of RNA sequencing data using R and Bioconductor. Nat Protoc. 2013;8(9):1765-86. doi:10.1038/Nprot. 2013.099.

42. Love MI HWaAS. Moderated estimation of fold change and dispersion for RNA-seq data with DESeq2. Genome Biol. 2014;15:550.

43. Law CW, Chen YS, Shi W, Smyth GK. Voom: precision weights unlock linear model analysis tools for RNA-seq read counts. Genome Biol. 2014;15(2):R29. doi:Artn R29. doi:10.1186/Gb-2014-15-2-R29.

44. Robinson MD, Smyth GK. Small-sample estimation of negative binomial dispersion, with applications to SAGE data. Biostatistics. 2008;9(2):321-32. doi:10.1093/biostatistics/kxm030.

45. Rapaport F, Khanin R, Liang Y, Pirun M, Krek A, Zumbo P, et al. Comprehensive evaluation of differential gene expression analysis methods for RNA-seq data. Genome Biol. 2013;14(9):R95. doi:10.1186/gb-2013-14-9-r95.

46. Cameron AC, Trivedi PK. Regression analysis of count data. Second edition. ed. Econometric society monographs, vol no 53. 2013.

47. White JR, Nagarajan N, Pop M. Statistical methods for detecting differentially abundant features in clinical metagenomic samples. PLoS Comput Biol. 2009;5(4):e1000352. doi:10.1371/journal.pcbi.1000352.

48. Connolly SR, Dornelas M, Bellwood DR, Hughes TP. Testing species abundance models: a new bootstrap approach applied to Indo-Pacific coral reefs. Ecology. 2009;90(11):3138-49.

49. Cheung YB. Zero-inflated models for regression analysis of count data: a study of growth and development. Stat Med. 2002;21(10):1461-9. doi:10.1002/sim.1088.

50. Holmes I, Harris K, Quince C. Dirichlet multinomial mixtures: generative models for microbial metagenomics. PLoS One. 2012;7(2):e30126. doi:10.1371/journal.pone.0030126

51. Auer PL, Doerge RW. Statistical design and analysis of RNA sequencing data. Genetics. 2010;185(2):405-16. doi:10.1534/genetics.110.114983.

52. Yu D, Huber W, Vitek O. Shrinkage estimation of dispersion in Negative Binomial models for RNA-seq experiments with small sample size. Bioinformatics. 2013;29(10):1275-82. doi:10.1093/bioinformatics/btt143.

53. Soneson C, Delorenzi M. A comparison of methods for differential expression analysis of RNA-seq data. BMC Bioinformatics. 2013;14:91. doi:10.1186/1471-2105-14-91.
54. Fierer N, Lauber CL, Zhou N, McDonald D, Costello EK, Knight R. Forensic identification using skin bacterial communities. Proc Natl Acad Sci U S A. 2010;107(14):6477-81. doi:10.1073/pnas.1000162107.

55. Kennedy K, Hall MW, Lynch MD, Moreno-Hagelsieb G, Neufeld JD. Evaluating bias of illumina-based bacterial 165 rRNA gene profiles. Appl Environ Microbiol. 2014;80(18):5717-22. doi:10.1128/AEM.01451-14.

56. Salter SJ, Cox MJ, Turek EM, Calus ST, Cookson WO, Moffatt MF, et al. Reagent and laboratory contamination can critically impact sequence-based microbiome analyses. BMC Biol. 2014;12:87. doi:10.1186/s12915-014-0087-z.

57. Lozupone C, Knight R. UniFrac: a new phylogenetic method for comparing microbial communities. Appl Environ Microbiol. 2005;71(12):8228-35. doi:10. 1128/AEM.71.12.8228-8235.2005.

58. Lozupone CA, Hamady M, Kelley ST, Knight R. Quantitative and qualitative beta diversity measures lead to different insights into factors that structure microbial communities. Appl Environ Microbiol. 2007;73(5):1576-85. doi:10. 1128/AEM.01996-06.

59. Bray JR, Curtis JT. An ordination of the upland forest communities of southern Wisconsin. Ecol Monogr. 1957;27(4):326-49.

60. Fernandes AD, Reid JN, Macklaim JM, McMurrough TA, Edgell DR, Gloor GB. Unifying the analysis of high-throughput sequencing datasets: characterizing RNA-seq, $16 \mathrm{~S}$ rRNA gene sequencing and selective growth experiments by compositional data analysis. Microbiome. 2014;2:15. doi:10. 1186/2049-2618-2-15.

61. Anderson MJ, Walsh DCI. PERMANOVA, ANOSIM, and the Mantel test in the face of heterogeneous dispersions: what null hypothesis are you testing? Ecol Monogr. 2013;83(4):557-74.

62. Lauber CL, Hamady M, Knight R, Fierer N. Pyrosequencing-based assessment of soil $\mathrm{pH}$ as a predictor of soil bacterial community structure at the continental scale. Appl Environ Microbiol. 2009;75(15):5111-20. doi:10.1128/AEM.00335-09.

63. Costello EK, Lauber CL, Hamady M, Fierer N, Gordon Jl, Knight R. Bacterial community variation in human body habitats across space and time. Science. 2009;326(5960):1694-7. doi:10.1126/science.1177486.

64. Caporaso JG, Lauber CL, Costello EK, Berg-Lyons D, Gonzalez A, Stombaugh J, et al. Moving pictures of the human microbiome. Genome Biol. 2011; 12(5):R50. doi:10.1186/gb-2011-12-5-r50.

65. JN Paulson MP, HC Bravo. metagenomeSeq: Statistical analysis for sparse high-throughput sequencing. Bioconductor package: 1.11.10 ed. 2013.

66. Carcer DA, Denman SE, McSweeney C, Morrison M. Evaluation of subsampling-based normalization strategies for tagged high-throughput sequencing data sets from gut microbiomes. Appl Environ Microbiol. 2011;77(24):8795-8. doi:10.1128/AEM.05491-11.

67. La Rosa PS, Brooks JP, Deych E, Boone EL, Edwards DJ, Wang Q, et al. Hypothesis testing and power calculations for taxonomic-based human microbiome data. PLoS One. 2012;7(12):e52078. doi:10.1371/journal.pone.0052078.

68. Chen J, Li H. Variable selection for sparse Dirichlet-multinomial regression with an application to microbiome data analysis. Ann Appl Stat. 2013;7(1). doi:10.1214/12-AOAS592.

69. Palarea-Albaladejo J. aJAM-FJA. ZCompositions-R package for multivariate imputation of left-censored data under a compositional approach. Chemom Intel Lab Syst. 2015;143:85-96.

70. Caporaso JG, Lauber CL, Walters WA, Berg-Lyons D, Huntley J, Fierer N, et al. Ultra-high-throughput microbial community analysis on the Illumina HiSeq and MiSeq platforms. ISME J. 2012;6(8):1621-4. doi:10.1038/ismej.2012.8.

71. Piombino P, Genovese A, Esposito S, Moio L, Cutolo PP, Chambery A, et al. Saliva from obese individuals suppresses the release of aroma compounds from wine. PLoS One. 2014;9(1):e85611. doi:10.1371/journal.pone.0085611.

72. Caporaso JG, Lauber CL, Walters WA, Berg-Lyons D, Lozupone CA, Turnbaugh PJ, et al. Global patterns of 16S rRNA diversity at a depth of millions of sequences per sample. Proc Natl Acad Sci U S A. 2011;108 Suppl 1:4516-22. doi:10.1073/pnas.1000080107.

73. Kaufman L. RP. Finding groups in data: an introduction to cluster analysis. Hoboken: JohnWiley \& Sons; 1990.

74. Reynolds A, Richards G, Iglesia B, Rayward-Smith V. Clustering rules: a comparison of partitioning and hierarchical clustering algorithms. J Math Model Algorithms. 2006;5:475-504.

75. Colwell RK, Chao A, Gotelli NJ, Lin SY, Mao CX, Chazdon RL, et al. Models and estimators linking individual-based and sample-based rarefaction, extrapolation and comparison of assemblages. J Plant Ecol-Uk. 2012;5(1): 3-21. doi:10.1093/Jpe/Rtr044.

76. Witten DM. Classification and clustering of sequencing data using a Poisson model. Ann Appl Stat. 2011;5(4):2493-518. doi:10.1214/11-Aoas493. 
77. Anderson MJ. A new method for non-parametric multivariate analysis of variance. Austral Ecol. 2001;26(1):32-46. doi:10.1111/J.1442-9993. 2001.01070.Pp.X.

78. Benjamini $Y$, Hochberg Y. Controlling the false discovery rate: a practical and powerful approach to multiple testing. J Roy Stat Soc B Met. 1995;57(1):289-300.

79. Kim BS, Margolin BH. Testing goodness of fit of a multinomial model against overdispersed alternatives. Biometrics. 1992;48:711-9.

80. Team RC. R: a language and environment for statistical computing. Vienna, Austria: R Foundation for Statistical Computing; 2014

81. Gentleman RC, Carey VJ, Bates DM, Bolstad B, Dettling M, Dudoit S et al. Bioconductor: open software development for computational biology and bioinformatics. Genome Biol. 2004;5(10). doi: Artn R80. 10.1186/Gb-2004-5-10-R80.

82. Vazquez-Baeza Y, Pirrung M, Gonzalez A, Knight R. EMPeror: a tool for visualizing high-throughput microbial community data. GigaScience. 2013; 2(1):16. doi:10.1186/2047-217X-2-16.

Submit your next manuscript to BioMed Central and we will help you at every step:

- We accept pre-submission inquiries

- Our selector tool helps you to find the most relevant journal

- We provide round the clock customer support

- Convenient online submission

- Thorough peer review

- Inclusion in PubMed and all major indexing services

- Maximum visibility for your research

Submit your manuscript at www.biomedcentral.com/submit 